\title{
DER ISLAM UND DIE WESTLICHEN IDEOLOGIEN: DIE IDEOLOGISCHE VERWIRRUNG DER ELITEN IN DER ISLAMISCHEN WELT
}

Interne soziale Krisen breiten sich in der islamischen Welt rasch aus. Die Ursachen dieser Entwicklung können von westlichen Wissenschaftlern noch nicht tiefgreifend erforscht werden, weil Sprach- und Mentalitätsbarrieren eine objektive Analyse erschweren. Hinzu kommt, daß aufgrund bestimmter ökonomischer Interessen des Westens die Massenbewegungen in der islamischen Welt mit Skepsis, ja mit Ignoranz betrachtet werden.

Die Revolution der islamischen Massen im Iran überraschte westliche Politiker ebenso wie westliche oder westlich-orientierte Forscher. Eine Revolution mit Hilfe des Islam gegen Ende des 20. Jahrhunderts hatte sich niemand im Westen vorstellen können. Die iranischen Ereignisse werden als ein neues Phänomen aufgefaßt. Man vergaß, daß noch zu Beginn dieses Jahrhunderts der Islam den Hauptantrieb in den Kämpfen gegen Kolonialismus geliefert hatte; so z. B. in Saudi-Arabien. Dort hatte Abdel Aziz ibn Abdar Rahman al Saud (im Westen als ibn-Saud bekannt) mit Hilfe des Islam Arabiens Stämme in einem Staat vereinigt und die Kolonisatoren vertrieben. Er und der ,,Wahhabismus“, dessen „Imam“ er war, dienten als Modell für islamische Bewegungen und Befreiungskämpfe. Den ,Senussis“ in Libyen gelang es, die islamischen Massen gegen fremde Kolonisatoren ebenso zu mobilisieren wie der „Mahdismus“ im Sudan. Der Islam entwickelte eine ungeheure Dynamik, die sie im Laufe der Jahrhunderte, insbesondere unter fremden Herrschern, verloren hatte. Die jüngsten islamischen Experimente von Libyen und insbesondere vom Iran haben die Weltöffentlichkeit auf den Islam und seinen Einfluß auf die islamischen Massen aufmerksam gemacht.

Dies führte jedoch fälschlicherweise dazu, daß man im Westen versuchte, die euro-amerikanische Offentlichkeit über den Islam als Religion aufzuklären. Die Literatur über den Islam expandierte in einem ungewöhnlichen Ausmaß. Damit gehen jedoch die euro-amerikanischen Medien an einem zentralen Punkt vorbei: Nicht der Islam als Religion ist ein neues Phänomen, sondern sein überraschender Einfluß auf die islamischen Massen heute. Dieser wiederum ist mit der kulturellen, ideologischen, sozialen, psychologischen, ökonomischen und schließlich politischen Entwicklung der islamischen Welt eng verknüpft. Das heißt der revolutionierende Effekt des Islam bei den islamischen Massen ist nicht ein Produkt kurzfristiger Vorgänge, wie es schlechthin angenommen wird. Indem sich aber die westlichen Medien bisher darin erschöpften, den Informationsmangel des Westens über den Islam zu beseitigen, ließen sie unbeachtet, welche tiefgreifenden Veränderungen in den islamischen Völkern durch die oben genannten Entwicklungsfaktoren zustande gekommen waren.

\section{Rückblick auf die historische Entwicklung}

Der Islam verlor im Laufe der Jahrhunderte viel von seiner ungeheuren Dynamik der ersten Jahre. Diese Entwicklung ging parallel zur politischen Entmachtung der Araber. Die einst mächtigen und vereinten Araber zerfielen in fast autonome Stämme, Orte, Gilden, Orden, „,die alle ihre Energien auf die Erhaltung der eigenen Existenz richteten“" Sie gerieten lang-

1 Steppat, Fritz: Die arabische Welt in der Epoche des Nationalismus, in: Taeschner, Franz: Geschichte der arabischen Welt, Stuttgart, 1964, pp. 178-236, p. 179. 
sam unter die Herrschaft nicht arabischer Herrscher. Damit begann für den Islam die Verfallsperiode, die sich besonders unter der Herrschaft der Osmanen beschleunigte. Es war die Fragmentierung der Araber, die es den nomadischen Türken aus Zentralasien ermöglichte, die arabischen Staaten fast kampflos zu kolonisieren, wobei sie oft ein arabisches Fürstentum gegen das andere ausspielten. Der Islam entwickelte sich unter der Türken-Herrschaft zu einer Herrschaftsideologie ${ }^{2}$. Allmählich verkümmerte der Islam. Er konnte sich den sozio-ökonomischen Entwicklungen nicht mehr anpassen und büßte an seiner Durchsetzungskraft ein. Die Forschung hatte im osmanischen Reich aufgehört; die ,Ulema“" (Religionsgelehrte) befaßten sich nur noch mit rein theologisch-herrschaftsideologischen Fragen. Der Islam verlor so die ihm innewohnende Dynamik zur Erneuerung. ,,Die Herrscher, die meist nicht arabischer Herkunft waren, wurden hingenommen, da sie sich zum Islam bekannten. Die vermittelnden und verneinenden Funktionen lagen bei den Ulema; aber wenn diesen auch das Recht zustand, die Herrscher zu kritisieren, so fügten sie sich doch im allgemeinen der Macht, und wenn sie auch für die Einheit der islamischen Religion und Kultur sorgten, so geschah das auf einem Stillstandsniveau. Im späten islamischen Mittelalter wirkten keine Kräfte für einen wissenschaftlichen oder sozialen Fortschritt. “3

Bei diesem Verfallsprozeß haben die Leitbilder in der Erziehung eine wichtige Rolle gespielt; während sich im Westen die Erziehung und Ausbildung immer stärker auf schöpferische Entfaltung und logisches Denken ausrichtete, erstarrte sie in der islamischen Welt in der Rekapitulierung religiöser Bücher, insbesondere des Korans und des Hadis. Diese Erziehungslinie wurde auch in der familiären Erziehung verfolgt. Die falsche Interpretation des Schicksalsglaubens hatte sich derart unter dem Einfluß von Orden, insbesondere unter den SufiOrden, verbreitet, daß man durchaus von einer Erziehung zur Faulheit sprechen konnte, eine Entwicklung, die in der islamischen Welt noch weitgehend anhält. Solange Gott, der Allmächtige, alles für den einzelnen Muslimen regelte, brauchte man sich um nichts mehr zu bemühen. "The Arabs believe in God and rely on him to do everything for them."4 . Die religiösen Orden haben diese Einstellung zum Leben gefördert. „,Die islamische Mystik hatte durch die Sufi-Orden starken Einfluß auf die breiten Volksmassen gewonnen. Aber während der Sufismus einst die Religion durch Verinnerlichung bereichert hatte, wirkte er sich nun schon lange vor allem im Drang zur Abkehr vom Diesseits, zur Verachtung irdischer Leistungen aus, wenn er nicht überhaupt in blindem Aberglauben versank. " 5 Körperliche Anstrengung und das Streben nach materiellen Gütern wurden allgemein verpönt und trugen dazu bei, daß noch heute die konservativen islamischen Massen sie verachten. Wer sich permanent anstrengt, steht im Verdacht, er wolle die göttliche Entscheidung bezweifeln. Hinzu kam der spezifisch-nomadische Einfluß der Türken mit der Verherrlichung des Soldatentums, das heißt der Gewalt, und der sinnlichen Liebe. Dies führte konsequenterweise zu verheerenden sozio-ökonomischen Folgen, die noch in der islamischen Welt herrschen. Die Erziehungsleitbilder förderten die Trägheit allgemein so, daß man den wenigen, die sich von der Religion emanzipierten, gleich ob Herrscher, Parlamentarier oder Präsidenten, bedingungslos vertraut, ohne an Form und Inhalt ihrer Herrschaft Kritik zu üben. Es ist daher nicht verwunderlich, daß die Zahl derer, die Sozialkritik in bildlicher Kunst oder Literatur vortragen könnten, auch äußerst klein geblieben ist. Der immense politische Druck schafft oft unüberwindliche Barrieren, an denen jegliche Kritik scheitern muß. Ein Zustand, der sich weitgehend von westlichen Praktiken unterscheidet.

\footnotetext{
2 Cf. Rodinson, Maxime: Islam und Kapitalismus, Frankfurt a. M., 1971.

3 Steppat, a. a. O., p 179

4 Events No. 25, v. 9. 9. 1977 , p. 13.

5 Steppat, a. a. O., p. 179
} 


\section{Die Kluft zwischen den islamischen Ländern und dem Westen}

Im Laufe der Zeit vertiefte der politische Druck in islamischen Ländern auch die Kluft in der Bildung, die zwischen den arabisch-islamischen Ländern und den fortgeschrittenen Staaten noch existiert. Diese Entwicklung führte dazu, daß nötige Informationen und Kenntnisse den arabisch-islamischen Völkern vorenthalten wurden und noch immer blockiert werden. Eine der wichtigsten Ursachen der sozio-ökonomischen Misere der arabisch-islamischen Länder ist jedoch der Bildungsrückstand. In der Türkei z. B. führte die übertriebene Angst vor dem Kommunismus dazu, daß Volkshäuser und Dorfinstitute, die eine wichtige Erziehungsfunktion in der kemalistischen Türkei hatten, geschlossen werden mußten. Sie spielten aber besonders in der Vermittlung moderner technischer landwirtschaftlicher Methoden eine entscheidende Rolle. Eine der Hauptursachen für die landwirtwirtschaftliche Stagnation in vielen arabisch-islamischen Ländern ist noch immer der Mangel an modernen landwirtschaftlichen Kenntnissen.

Die sozio-ökonomische Misere in der arabisch-islamischen Welt ist das Produkt einer langen Entwicklung. Insbesondere die Araber wurden im osmanischen Reich vernachlässigt und lediglich als Steuerzahler betrachtet. Ihr Reichtum wurde in den Kriegszügen der Pforte verschleudert. Vergleicht man die Araber mit den Israelis, besonders auf dem Gebiet der Bildung und Erziehung, so haben die Araber sehr viel nachzuholen. Während die europäischen Juden sich eine moderne Erziehung aneignen konnten, verkümmerten die Araber im stagnierenden osmanischen Erziehung- und Bildungssystem. Daher wäre es falsch, T. H. Lawrence, bekannt als Lawrance von Arabien, zu folgen, der die Araber in seine klischeehaften Vorstellungen hineinpackt: "Their convictions were by instinct, their activities intuitional."6 Damit glaubte Lawrence einen arabischen Charakterzug erkannt zu haben. Dabei handelt es sich um etwas, das erziehungsbedingt ist und keineswegs angeboren ${ }^{7}$. In einer allgemeinen Stagnationsphase sind alle Bereiche betroffen. Unter anderen sozialen Voraussetzungen blühte die arabische Kunst und Wissenschaft. Zur Zeit der Abbasiden oder unter den Almoraviden und Almohaden in Spanien herrschte lebhafte geistige Auseinandersetzung. Diese weltoffene Atmosphäre führte bemerkenswerterweise zur Gründung der ersten eigentlichen Universitäten im westlichen Sinne. Arabische Forscher waren Lehrer des Abendlandes wie z. B. in der Algebra, der Medizin, der Chemie (siehe Wörter wie ,Alkohol"). Arabische Baumeister wagten kühnste Architekturen, arabische Seeleute entwickelten die Navigation mit Vermessungsinstrumenten und wagten sich in fernste Weltgegenden, und arabische Geographen schufen umfassende Kartenwerke. Historiker brachten Beschreibungen ferner Gesellschaften.

In einer Phase allgemeiner gesellschaftlicher Stagnation verlor jedoch der Islam unter türkischen Herrschern, die keine Kritik vertrugen, seinen Elan und verpaßte dann - in der späteren Konfrontation mit der Technik des Westens - die Anpassung an die sich wieder beschleunigenden sozio-ökonomischen Entwicklungen. Die Gesellschaft versagte bei dem Versuch, auftauchende zeitbedingte Probleme mit Hilfe der Religion und Interpretation der Koranverse zu lösen. ,,In der islamischen Theologie galt das Tor der freien Forschung als geschlossen; den Religionsgelehrten (Alim, Plural, Ulema) war nur die Aufgabe geblieben, einen unveränderlichen Wissensbestand von Generation zu Generation weiterzureichen."8 Mit dem Erlöschen der politischen Dynamik erlosch auch jegliche geistige und sonstige Ak-

6 Lawrence, T. E.: Seven Pillars of Wisdom, London, 1946, p. 37

7 Lawrence, wie viele seiner westlichen Zeitgenossen (Reisende oder ,,Forscher“ insbes. aus England) perpetuierten durch solche verkehrte Feststellungen ein verdrehtes Araberbild, cf. hierzu auch Said, Edward: Orientalism, London und Henley, 1978.

8 Steppat, a. a. O., p. 178 
tivität. Während in Europa neue Konfessionen (Protestantismus, Pietismus) eine neue Dynamik hervorriefen, die wiederum zur technisch-industriellen Revolution in Europa führte ${ }^{9}$, erstarrte die islamische Welt durch das Fehlen neuer Ideen. In Europa hatte jedoch mit der Renaissance und der Hinwendung zu naturwissenschaftlicher Forschung ein Prozeß begonnen, der sich dann immer mehr beschleunigte. Die Forschung lieferte nun Impulse, die bislang die Religion lieferte. Sie wurde somit zum Motor des sozialen, ökonomischen und politischen Wandels. Bei nachlassender Forschung müßte auch heute die westliche Gesellschaft stagnieren, so wie es die islamische Welt seit Jahrhunderten tut.

Diese Zusammenhänge werden jedoch in den arabisch-islamischen Ländern nicht erkannt. Ihre Regierungen sehen nach wie vor in freier Forschung eine Gefahr für ihre Machtstellung. Sie blockieren daher das Geistesleben in ihren Staaten und perpetuieren so die sozio-ökonomische Misere und halten ihre Länder langfristig in Abhängigkeit von fremden Ideen, die weder den Bedürfnissen noch den Voraussetzungen der Massen in diesen Staaten entsprechen und deswegen zum Scheitern verurteilt sind.

\section{Die Ubernahme westlicher Entwicklungskonzepte}

Zunächst kapitalistische, später sozialistische Konzepte fanden Zugang in die islamische Welt - insbesondere durch die Unterstützung von Kolonial-Eliten (Armee und Bürokratie). Bei den religiösen, traditionellen Massen, die den Sinn des Lebens durch die Religion und Tradition gegeben sahen, erweckten sie jedoch durch ihre extreme Diesseitsbezogenheit Abscheu; denn, so Kolakowski: ,,Es gibt keinen Sinn in der Welt, keine Pflichten, keineSünde. Nichts hat einen Sinn. Sinn ist von uns willkürlich oder durch Glauben der Welt, der Geschichte aufoktroyiert - empirisch ist er weder beweisbar noch deduzierbar. "10 Den Sinn des Lebens sieht der Muslim nicht im Streben nach materiellen Gütern: ,, Wir sind ein Volk der Askese. Wir verachten das Angebot der Welt, und wie wir unsere wenigen Tage auf Erden verbringen, interessiert uns nicht. "11 Den Sinn des Lebens hat der Koran bereits geklärt. Danach ist die Welt für den Muslim in Ordnung. Das vorläufige und unangenehme Leben im Diesseits wird als Vorbereitungsphase auf das ewige, paradiesische Leben aufgefaßt. Unter diesen Umständen wurde konsequenterweise mehr Zeit für religiöse Pflichten eingeräumt. Der im Koran verankerte Lohn für das Streben im Diesseits geriet so in Vergessenheit. Er war nicht so attraktiv wie die Belohnung im Jenseits. Verschiedene Hinweise verdeutlichen diesen paradoxen Zustand, wie das folgende arabische Sprichwort: ,Er baute (ein Haus), er ging (starb) und ließ es auf dieser Erde zurück. "Daraus folgt: Wenn man alles zurücklassen muß, und das irdische Leben so kurz und vorläufig ist, warum sollte man sich dann erst bemühen? Die religiösen Orden hatten die fundamentale Bescheidenheit des Orientalen - die auch vor dem Islam existierte - geschickt für ihre Jenseits-Propaganda ausgenützt. Dabei mißachteten sie die Koran-Suren, die z. B. das Arbeiten für das Diesseits als eine gottgefällige Aufgabe fordern. Eine konsum- und daher leistungsorientierte Sinngebung des Lebens, wie Marx es mit der auf Wachstum zielenden Formulierung (mehr produzieren und mehr verbrauchen) zusammenfaßte, hat für den Muslimen keine Relevanz. Ihn können daher we-

\footnotetext{
9 In England und Holland gab z. B. der Calvinismus diesen neuen Mächten den Schwung, sich gegen Spanien durchzusetzen und über die Meere zu fahren. Von hier direkte Verbindung auch zum Kapitalismus in den USA. Cf. Weber, Max: Gesammelte Aufsätze zur Religionssoziologie, Bd. I, Tübingen, 1972; ders.: Wirtschaft und Gesellschaft. Grundriß der verstehenden Soziologie, Tübingen, 1976.

10 Marxismus ist das Opium des Volkes. Ein Gespräch mit Leszek Kolakowski anläßlich des Friedenspreises des Deutschen Buchhandels, in: Die Zeit, Nr. 43, v. 14. 10. 1977.

11 Johansen, Baber: Muhammad Husein Haikal:Europa und der Orient im Weltbild eines ägyptischen Liberalen, Beirut, 1967, p. 15.
} 
der der Kapitalismus noch der Kommunismus bzw. der Sozialismus ansprechen. Die Religion ist die einzige Quelle der Motivation. Die islamischen Massen wären daher auch dann nicht zu bewegen, wenn man mit einer gezielten Propaganda versuchen würde sie davon zu überzeugen, daß das paradiesische Leben mit Hilfe materieller Güter im Diesseits erzielt werden könnte. Hinzu kommt, daß die islamische Welt die negativen Folgen einer leistungsund konsumorientierten Gesellschaft sieht, die man in eigenen Ländern vermeiden möchte. Das Streben nach besserer Lebensqualität gilt daher als erstrebenswerter. Für mehr Freizeit ist der Muslim bereit, auf mehr materielle Güter zu verzichten. Die fundamentale Bescheidenheit, die ihm innewohnt, macht ihn nur für das Notwendigste ansprechbar: z. B. medizinische Versorgung, bessere Bildungschancen für seine Kinder und vor allem ausreichende Versorgung mit billigen Lebensmitteln und den wenigen Kleidungsstücken für den täglichen Bedarf. Er stellt keine hohen Ansprüche an das Leben in Form von Konsumgütern der Luxusklasse und steht oft fassungslos der ,,Konsumgier“ des Westerners gegenüber ${ }^{12}$.

Die kulturelle Dependenz ${ }^{\mathbf{1 3}}$ blockiert jedoch die Auf wertung islamischer Werte. Sie sind einem permanenten, externen Destruktionsprozeß ausgesetzt. Auch von den kolonisierten Eliten werden sie bekämpft und sind unter politischem, ökonomischem und sozialem Druck. "While the elite elaborated the secular ideas of Arab Nationalsm, the masses remained fixed in islamic traditionalism." 14 Das heißt die Massen haben weder die importierten säkularen Ideen noch den damit verbundenen arabischen Nationalismus ganz akzeptieren können. Wie kann jedoch der arabische Nationalismus verteidigt werden, wenn die Volksbasis fehlt? "There could only be a nation in the real sense, if the people had something to defend", so Musa al-Alami15. Vom jüngsten iranischen Beispiel abgesehen, waren die islamischen Massen bisher nicht zu mobilisieren (siehe die kemalistische Türkei). Unter säkularen Regimen des Kemalismus oder Nasserismus blieb den islamischen Massen nichts anderes übrig, als respektvoll aber apathisch abzuwarten. Weder Kemalismus noch Nasserismus oder Baathismus als Konglomerate aus kapitalistisch-sozialistischen Elementen vermochten den islamischen Massen eine Alternative zum Islam zu bieten. Die Führungs-Eliten der islamischen Welt übersahen, daß westliche Konzepte schwer zu praktizieren sind, das heißt von arabisch-islamischen Völkern schwer zu verinnerlichen sind, weil sie nicht ein Produkt aus dem Potential der Region sind.

\section{Die Identitätskrise und die Suche nach dem ,,eigenen Weg““}

In einem Zustand, in dem alle Normen umgekippt sind, herrscht Anomie. Den Menschen fehlt es an Orientierungshilfen. Sie können von keiner Regelmäßigkeit im Leben mehr ausgehen, die ihnen die nötige Ruhe und Sicherheit verleihen würde. Brezezinski kennt diese Problematik aufgrund seiner Erfahrungen in den USA. Er weiß, was es bedeutet, wenn Werte zerstört werden, ohne sie wirklich zu ersetzen, wenn Chaos als Folge davon ausbricht und die Menschen total verunsichert werden: Ein Zustand, den man in der islamischen Welt häufig beobachten kann. Dort, wo westliche Ideologien islamische Werte zerstören, verursachen sie ein Vakuum und führen so zum Identitätsbruch. Die islamischen Massen wollen

12 Die Bescheidenheit des Muslimenkönnte als Beispiel den Industrienationen dienen, um ihr Konsumverhalten zu korrigieren und zur alternativen Lebensform zurückzufinden. Dies würde nicht nur zur Lösung u. a. derökonomischen und psychologischen Probleme beitragen, sondern auch die existente Kluft zwischen, ,sehr arm" und ,,sehr reich" reduzieren, d. h., daß diese Polarisierung allmählich einem Mittelweg ausweichen könnte. Dadurch könnte der Nord-Süd-Konflikt langsam abgebaut werden.

13 Onder, Zehra: Zur kulturellen Dependenz der ,Dritten Welt“, in: Neue Politik, 24. Jg./X, 15. Oktober 1979, pp. 50-59.

14 Sharabi, Hisham: Unity, Disunity and Fragmentation in the Arab World, in: Said, Edward/Suleiman, Fuad (Hg.): The Arabs Today. Alternatives for Tomorrow, Columbus, 1973, pp. 137-142, p. 138.

15 Hourani, Albert: Arabic Thought in the Liberal Age 1798-1939, London, Oxford, New York, 1970, p. 355. 
einerseits am aufgezwungenen ,,Prozeß der Modernisierung “ nicht teilnehmen, andererseits hat die Führung für sie kein Verständnis. Auf Dauer ist daher die Paralysierung des Staatslebens unvermeidlich. Die blutigen Auseinandersetzungen wie jüngst im Iran und gegenwärtig in der Türkei zwischen Eliten und Volk sind ein Indiz dafür. ,,Eine Gesellschaft, die an nichts glaubt", so Brezezinski, ,,ist eine Gesellschaft in Auflösung" "16. Die Ereignisse von Guayana mit dem Massenmord von Amerikanern im November 1978 unterstützen seine These im Hinblick auf die Vereinigten Staaten. Der amerikanische ,Materialismus und Vulgarität", hebt Brezezinski hervor, ,, unterminiert den traditionellen Glauben, ohne eine philosophische Alternative von Bedeutung an dessen Stelle zu setzen"17. Bereits vor der Wahl Carters zum Präsidenten wies er darauf hin: ,Was die Amerikaner heutzutage brauchen, ist nicht charismatische Führung; sie brauchen spirituelle Führung. "18

Eine spirituelle Führung benötigen die islamischen Völker mehr denn je. Danach würden die islamischen Massen das nötige Selbstbewußtsein und die Sicherheit wiedererlangen. Die Gründung einer symbolischen spirituellen ,Zentrale“ ist auch unter diesem Aspekt von Bedeutung. Nur sie wäre imstande, alle islamischen Massen zu erfassen und auf einen sozio-ökonomischen Wandel vorzubereiten. Die Existenz eines Kalifats hätte die notwendige Legitimation dazu und entspräche ferner den vorhandenen Realitäten. Diese Notwendigkeit wurde bereits von Kadhafi, wie vor ihm von Abdel Aziz oder dessen Sohn Faisal erkannt. Die Ziele Saudi-Arabiens und Libyens sind im Kern gleich. Der entscheidende Unterschied liegt darin: Während Saudi-Arabien eine langsame, das heißt langfristig angelegte sozio-ökonomische Evolution anstrebt, konzentriert sich Libyen auf die Lösung dringender Probleme, in die das Land - bzw. die gesamte islamische Welt - durch die Kolonialmächte hineingebracht wurde.

Beide Länder antworten auf die wissenschaftliche und wirtschaftliche Herausforderung des Westens mit dem Islam. Saudi-Arabien ist ein klassischer Versuch eines islamischen Modells. Libyen und der Iran sind die jüngsten islamischen Experimente, denen andere folgen werden. Die Bemühungen sind um einen ,,eigenen islanischen Weg“ konzentriert. Saudi-Arabien verfolgt dabei die Strategie des islamischen Formalismus und kann daher zur Erneuerung des Islam kaum etwas beitragen bzw. als Modell dienen; Kadhafi hingegen praktiziert eine den islamischen Bedürfnissen angepaßte Entwicklungsstrategie: "An economic system of social justice which does not necessarily have to be either capitalist nor communist and does not prohibit the accumulation of wealth (linked with a progressive tax system) as long as it does not favour exploitation. Islam should be over all things, as it has always ruled over the economic, political and social life of the individual, of the family and of the national and international societies (of Moslems) alike."'19 Nach 400jähriger türkischer Herrschaft und anschließender Herrschaft der europäischen Mächte sind die Araber zum erstenmal seit Jahrhunderten imstande, einen eigenen Weg zu gehen. Von dieser ,,neuen Phase“ erhofft man für die islamischen Völker sehr viel. Dies ist tief im Bewußtsein der Araber verankert. Der Wunsch, einen ,,eigenen, islamischen Weg“" zu gehen, erreichte mit der islamischen Revolution im Iran einen Höhepunkt. Dabei will man sich vom Westen abgrenzen, dessen Techniken zwar benötigt werden, dessen Werte und Normen jedoch zugunsten der Wahrung der islamischen Identität ferngehalten werden müssen. Nur diese islamischen Werte können den externen Destruktionsprozeß stoppen und Millionen Muslimen in der ganzen Welt helfen, aus der Identitätskrise herauszukommen. Dadurch könnte auch eine wichtige

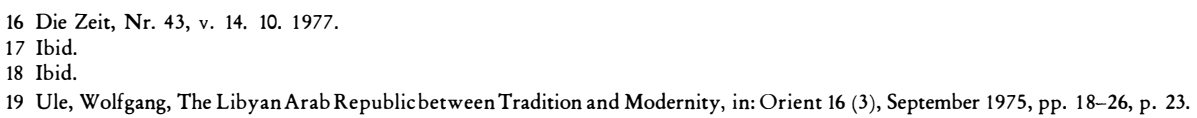


Barriere auf dem Wege zu schöpferischen Leistungen entfallen, die eine Entwicklung mit unabsehbaren Konsequenzen einleiten könnte. Dennoch sind die Kolonial-Eliten in der islamischen Welt, die sich an westlichen Ideologien (auch der Marxismus ist ein im Westen entstandenes Konzept) orientieren, nicht imstande, diese Zusammenhänge zu erkennen. Daher wird die Bedeutung des ,, islamischen Weges" für den Úbergang zur Moderne übersehen, die Dynamisierung der Massen für einen sozio-ökonomischen Wandel mit Hilfe des Islam verkannt. Auch Libyen verfolgt eine solche Strategie erst in Ansätzen.

Kadhafis , ,islamischer Weg“ stößt bei den Kolonial-Eliten auf Unverständnis. Er wird daher als Phantast oder Reaktionär abgestempelt. Die kulturelle Dependenz blockiert noch eine Versöhnung der Kolonial-Eliten mit den islamischen Massen und verursacht Spannungen zwischen ihnen. Die Politiker spüren, daß sie nicht länger die Bedürfnisse der Massen ignorieren können, daß die islamischen Massen innerlich angesprochen werden wollen. Nur dadurch können die Politiker ihre Machtstellung langfristig behaupten. Ein politisch-soziales Konzept, das den Islam in ihre Entwicklungsstrategie integriert, fehlt. Immer mehr breitet sich jedoch die Enttäuschung von den westlichen Ideologien unter den islamischen Massen aus. Westliche Ideologiekonglomerate wie der Kemalismus oder der Nasserismus konnten den Islam insbesondere auf dem Lande nicht verdrängen. Der Islam war zum Beispiel im türkischen Dorf stärker als die Regierung ${ }^{20}$. Kapitalistische oder sozialistische Elemente wirkten auf die Massen belastend. Dennoch versuchten zum Beispiel Kemal und seine Nachfolger in der Türkei, trotz des Volkes, das heißt gegen den Volkswillen zu regieren. Bei näherer Analyse erweisen sich solche ,,volksfremde Regierungen“ in der islamischen Welt als zu schwach, um sich langfristig behaupten zu können. Unter diesen Umständen breitet sich religiöse Solidarität in Form von zum Beispiel ,,Muslim-Bruderschaften“ (Ichwan) rasch aus. Die jüngsten Vorfälle in Syrien und davor in Ägypten haben uns diesen Sachverhalt deutlich vor Augen geführt. Diese Entwicklung kommt insbesondere Saudi-Arabien zugute. ,,Was vormals als ,Reaktion' galt, erscheint heute eher als Bewahrung echter Traditionen des Arabertums und läßt Saudi-Arabien ein hohes Maß an Achtung zukommen. "21 Saudi-Arabien lehnt die westlichen Ideologien - den Kapitalismus zumindest verbal - ab und versucht die islamischen Werte die in der islamischen Welt durch diese Ideologien zerstört worden sind, wiederzubeleben. Der Islam als ideologisches Fundament soll helfen, die ideologische Zerrissenheit innerhalb der islamischen Welt abzubaיen. Der Islam kann besonders die arabische Welt einen. "Islam retained many of their previous customs, adopting and polishing the best of them." 22

Sollte die ideologische Zerrissenheit der Araber abgebaut werden, so wird eine der folgenschweren Barrieren zwischen den Staaten der arabischen Welt und zugleich zwischen Eliten und Volk entfallen. Dies gilt auch für die gesamte islamische Welt. Ein solches Fundament kann eine arabische und eine islamische Einheit ermöglichen. Dadurch wird der Einfluß westlicher Ideologien in der arabisch-islamischen Welt verdrängt und eine eigenständige, aus dem Islam abgeleitete Identität gefördert. Dies ist notwendig, um den Selbstrespekt und das Selbstvertrauen wiederzuerlangen. Daraus kann sich eine neue, eigenständige Entwicklung aus dem Potential der Region anbahnen. Eine solche Entwicklung würde die arabisch-islamische Gesellschaft langfristig fundamental wandeln und eine Eigendynamik auslösen, die die Völker in eine Zukunft führen könnte, die ihren Voraussetzungen und Bedürfnissen entspricht. Das bedeutet nicht, daß alle westlichen Werte verworfen werden sollten. Hier kommt es insbesondere auf den Selektionsprozeß an, so daß die übernommenen Werte, die

20 Cf. Mardin, Serif: Din ve Ideoloji (Religion und Ideologie), Ankara, 1969.

21 Steinbach, Udo: Wohin entwickelt sich die arabische Welt?, in: Außenpolitik, Jg. 27 (1), 1976, pp. $51-61$, p. 54.

22 Smith, Donald Eugene (Hg.): Religion, Politics and Social Change in the Third World, New York, London, 1971 , p. 118. 
geistige Eigenständigkeit und damit die Identität dieser Völker nicht mehr in Frage stellen. „Zum wirklichen Leiden, zur Hölle, wird das menschliche Leben nur da, wo zwei Zeiten, zwei Kulturen und Religionen einander überschneiden. " 23 " Culture is the chief instrument in the creation of a people's identity. The assertion of a nation's personality corresponds in the cultural sphere to independence and sovereignity in the political sphere. A people want to be itself." 24

Westliche Entwicklungsmodelle sollten durchaus als Rohmaterial herangezogen werden, als Beispiel oder Lehrmodelle, nach deren Studium Entwicklungsstrategien aus dem eigenen Potential zu konzipieren sind. Der Islam ist die gegenwärtige Alternative zu westlichen Ideologien und ist imstande, die islamischen Massen zu mobilisieren. Auf diese Antriebskraft zu verzichten, hieße das Volkspotential ungenutzt lassen. Dieses Volkspotential kann in allen islamischen Ländern aktiviert werden. Die Integration der Massen in den Entwicklungsprozeß wird erst dadurch möglich. Der Islam würde darüber hinaus die ideologische Verwirrung in der islamischen Welt aufheben und den Individualismus vieler Muslimen neutralisieren.

\footnotetext{
23 Hesse, Hermann: Der Steppenwolf, Frankfurt a. M., 1978, p. 27 f.

24 Culture and Technology: Keys to National Identity and Development, an Interview with Mircea Malitza, in: UNESCO Features, No. $707 / 708$ (1976), pp. 6-10, p. 6.
} 


\section{Islam and Western Ideologies \\ By ZEHRA ÓNDER}

Islamic countries are at present in a crisis. This crisis has many dimensions: a cultural one (with its historical perspective), an ideological, a social, a psychological, an economic and also a political one. The Islamic revolution in Iran is the recent expression of this crisis. After centurylong influence of colonial powers the Islamic people are now on the way of indentity-finding. Islam as a religion is not new, but its dynamics of mobilization today. Under foreign predominance Islam in the course of history has lost very much from its dynamics and became, especially after the invasion of Turco-Tatarian people (nomadic tribes of Central Asian steppes) of Afghanistan, North-India, Iran, Asia-Minor and nearly all the Arab world (Ottoman empire) a power-ideology (Herrschaftsideologie). That development had devasting and lasting consequences on the Middle East, which was at that time culturally, socially and economically highly developed. The intellectual life in these countries was paralysed and paved the way for the later stagnation.

Parallel to this development, Europe experienced a huge cultural, ideological, social, technical, economical, military and political rise. This development widened the gap between the Middle East and Europe. While in Europe secular philosophies presented alternatives to religion, the Islamic world grew stiff through the lack of new impulses. To close the gap between occident and orient, the elites adopted Western concepts without any criticism and fell into cultural dependency. Islamic masses remained however without understanding of foreign concepts (capitalist, communist or conglomerates of those), because the values and norms were neither adequate to their presuppositions nor to their needs. The dualism as a result of this development on nearly all levels caused the identity-crisis. Only when Islam as an authentic instrument of development is generally accepted and developed, the ideological confusion within the Islamic-Arabic world on the one hand and the identity-crisis on the other hand, could be overcome.

\section{On Modern Education, Students and Social Change in the Islamic Middle East By BASSAM TiBI}

This paper starts from the thesis that modern education, inasmuch as it introduces new patterns of socialization, is one of the factors contributing to the dissolution of traditional social structures. Through modern education traditional societies are being westernized, i.e. traditional education which is largely limited to the memorization of learned sources is thus also being removed. Based on Gramsci's definition of the intellectual this paper differenciates between , learned“ and ,,intellectual“. The intellectual does not memorize like the traditional learned; rather, the intellectual's thinking is problem-oriented.

In Islamic societies westernized intellectuals have been the agents of social change since the 19 th century (the generation of Tahtawi). Yet, inasmuch as the oriental family still remains a very important agent of socialization also in transitional societies, the effects of the new patterns of socialization must be seen in a modified way. This paper develops the thesis, that modern education is introduced into an underdeveloped society which is lacking the infrastructure for the new institutions. This results in an only partial modernization, i.e. in a coexistence of modern and traditional elements in one and the same structure, a structure which must be defined as heterogeneous. 